\title{
A COMPARATIVE STUDY FOR THE EFFICIENCY OF THE PLATELET- RICH PLASMA AND HYALURONIC ACID IN TREATMENT OF TEMPOROMANDIBULAR JOINT OSTEOARTHRITIS
}

\author{
Hesham Fattouh*
}

\begin{abstract}
Objectives: The aim of this study is to compare the effect of Platelet-rich plasma (PRP) and Hyaluronic acid (HA) in management of Temporomandibular joint osteoarthritis (TMJ-OA)

Patients and methods: Among 46 patients who were diagnosed with TMJ-OA, 14 patients were treated with PRP, 17 patients were treated with HA and 15 patients were treated with arthrocentesis alone, evaluation of clinical improvement was done for each patient postoperatively at 6 and 12 months.
\end{abstract}

Results: After 6 months, there were no statistically significant differences in the mean changes between PRP and HA groups regarding the pain intensity value, joint sound and extent of maximum mouth opening, while at the end of the follow-up period, after 12 months, better clinical results were maintained in the group treated with PRP.

Conclusion: The application of PRP is more effective and remains for long term than HA injection TMJ-OA treatment.

KEYWORDS: TMJ - osteoarthritis - Platelet rich plasma - Hyaluronic acid

\section{INTRODUCTION}

Osteoarthritis (OA) is a chronic, complex and progressive disease which is characterized by pain, stiffness and loss of function of the affected joint ${ }^{(1)}$. Temporomandibular joint osteoarthritis (TMJ-OA) is one of most well-known disorders affecting the temporomandibular joint (TMJ), it is more frequent in females and its prevalence increases in relation to age. Many factors such as bruxism, uni-lateral chewing, hereditary factors and internal derangement are considered responsible for the development of TMJ-OA ${ }^{(2)}$. The diagnostic criteria for TMJ-OA are history of TMJ noise during function or movement upon examination or within the last 30 days together with crepitus at

\footnotetext{
* Lecturer of Oral and Maxillofacial Surgery, Faculty of Oral and Dental Medicine, Cairo University.
} 
movement during palpation, these clinical criteria should be adjunct with imaging to complete the diagnosis of TMJ-OA, computerized tomography (CT) has significantly superior sensitivity over magnetic resonance imaging (MRI) in diagnosis of $\mathrm{TMJ}_{-}-\mathrm{OA}^{(3)}$.

When aiming to restore the joint's asymptomatic status with minimal intervention, non-surgical management of TMJ-OA gives good and stable prognosis. However, when it fails, invasive surgical intervention (gap arthroplasty, with partial menisectomy, when required) is recommended ${ }^{(3)}$. TMJ arthrocentesis alone or arthrocentesis together with several intra-articular injections (e.g., plateletrich plasma, corticosteroids, hyaluronic acids, sodium hyaluronate) have been advocated for treatment of TMJ-OA, because of their high efficacy in improving jaw function and reducing pain levels via lavage the upper joint compartment, eliminating the inflammatory products, and aggressively manipulating the joint ${ }^{(4)}$.

In the treatment of degenerative pathology of the temporomandibular Joint, Platelet-rich plasma (PRP) exhibit anti-inflammatory, analgesic and antibacterial properties. PRP also restores intra-articular hyaluronic acid and balances joint angiogenesis; What's more, it provides a scaffold for stem cell migration and increases glycosaminoglycan chondrocyte synthesis ${ }^{(5)}$.

The injection of HA (Hyaluronic acid) after arthrocentesis has been postulated to decrease inflammation owing to the inherent anti-inflammatory properties of this agent and its ability to increase intra articular viscosity and lubrication ${ }^{(6)}$. Previous study suggested that it may be effective in inflammatory-degenerative disorders as well, especially if combined with a thorough joint lavage ${ }^{(7)}$.

The main aim of this study was to compare the effectiveness of PRP and HA injections following arthrocentesis in management of TMJ-OA.

\section{PATIENTS AND METHODS}

The following randomized prospective study was conducted to evaluate the efficiency of the PRP and HA in treatment of TMJ-OA, it was carried in accordance with international standards of quality for clinical trials, the Declaration of Helsinki in its revised version (Seoul, Korea, 2008), The study sample was derived from the population of patients who were presented to the Department of Oral and Maxillofacial Surgery, Cairo University. The inclusion criteria included patients with symptomatic TMJ-OA in one or the two joints that had no previous treatments (either conservative or surgical) for TMJ disorders. Diagnosis of TMJ-OA was done on clinical basis by the positive finding of at least two of the five following criteria: (1) pain upon palpation of the joint, (2) localized joint pain that increased upon loading and decreased on unloading, (3) localized pain in the affected joint upon passive and active jaw movement, (4) joint crepitating (accompanied with or without clicking), (5) limited maximum mouth opening (MMO) $(<35 \mathrm{~mm})^{(1)}$, the diagnosis was confirmed radiographically using CT by the presence of mild to severe degenerative changes. Patients were excluded if they have previous TMJ treatment or had inadequate data at the end of the follow-up period; also patients with hematologic or neurologic disorders, rheumatoid arthritis, inflammatory or connective tissue disease were excluded. All participants signed an informed consent agreement.

Patients were randomly assigned to one of the three following groups according to the planned line of treatment:

\section{Group I: (PRP group)}

Participants in the PRP group initially received arthrocentesis with $100 \mathrm{~mL}$ of Ringers lactate followed by $1 \mathrm{ml}$ PRP injection and then 2 consecutive PRP injections into the TMJ following intra-articular anaesthesia at a monthly interval. 


\section{Group II: (HA group)}

Participants in the HA group initially received arthrocentesis with $100 \mathrm{~mL}$ of Ringers lactate followed by $1 \mathrm{ml}$ of low molecular weight $\mathrm{HA}$ injection and then 2 consecutive HA injections into the TMJ following intra-articular anesthesia at monthly interval.

\section{Group III: (Control group)}

This group served as a control group for the first two groups, they received arthrocentesis with 100 $\mathrm{mL}$ of Ringers lactate only once.

No anti-inflammatory drugs were given to any group during the treatment period because such drugs may interfere with the healing process specially that initiated by the PRP injection, a gentle range of motion and liquid and/or semi-liquid eating was recommended post operatively.
The main outcome variables including: pain was measured using VAS (Visual Analogue Scale) evaluations, VAS has 2 ends: one end or 0 is marked as "no pain", and the other end or 10 is marked as "the worst imaginable" pain, MMO measured as the distance between the upper and lower incisors the patient could obtain without having any type of pain and it is expressed in millimeters, presence of any self-perceived pathologic noise with joint movement following scale: one end or 0 is marked as "no noise", and the other end or 10 is marked as "the worst imaginable noise". These data were recorded preoperatively and at intervals of 6 and 12 months postoperatively by the same surgeon, The surgeon is not blinded as to the treatment groups, also data regarding the age and gender of the patient were collected to assess the influences of demographic features on treatment effectiveness.

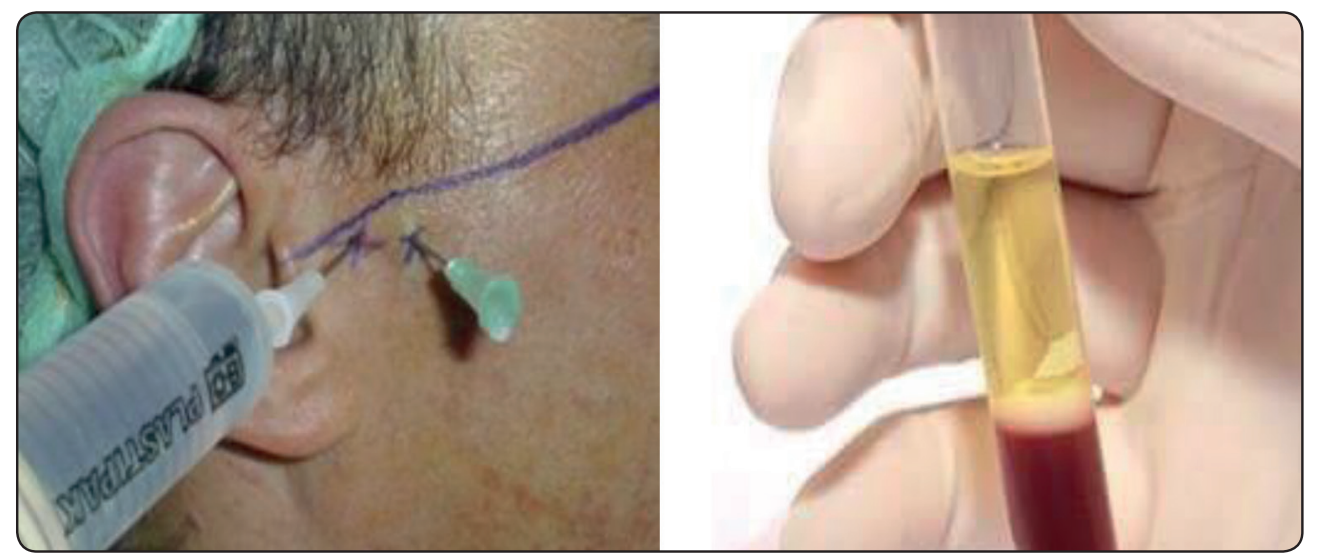

Fig. 1 (A) Arthrocentesis for a patient in the PRP group (B) The PRP extract used for injection in the TMJ

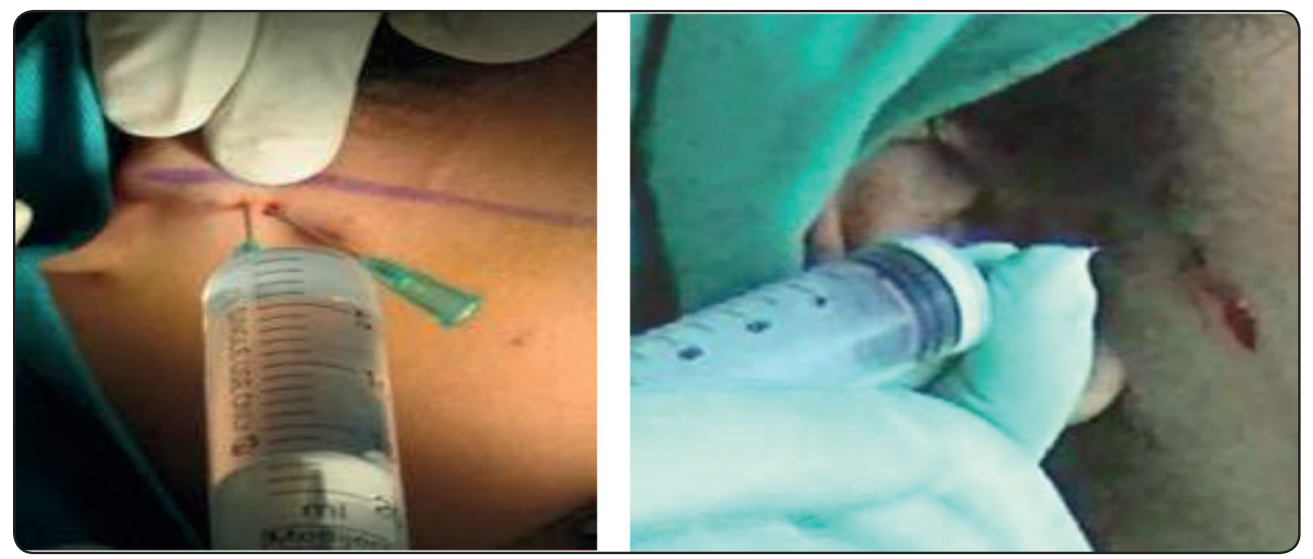

Fig. 2 (A) Arthrocentesis for a patient in the HA group (B)The HA injection 


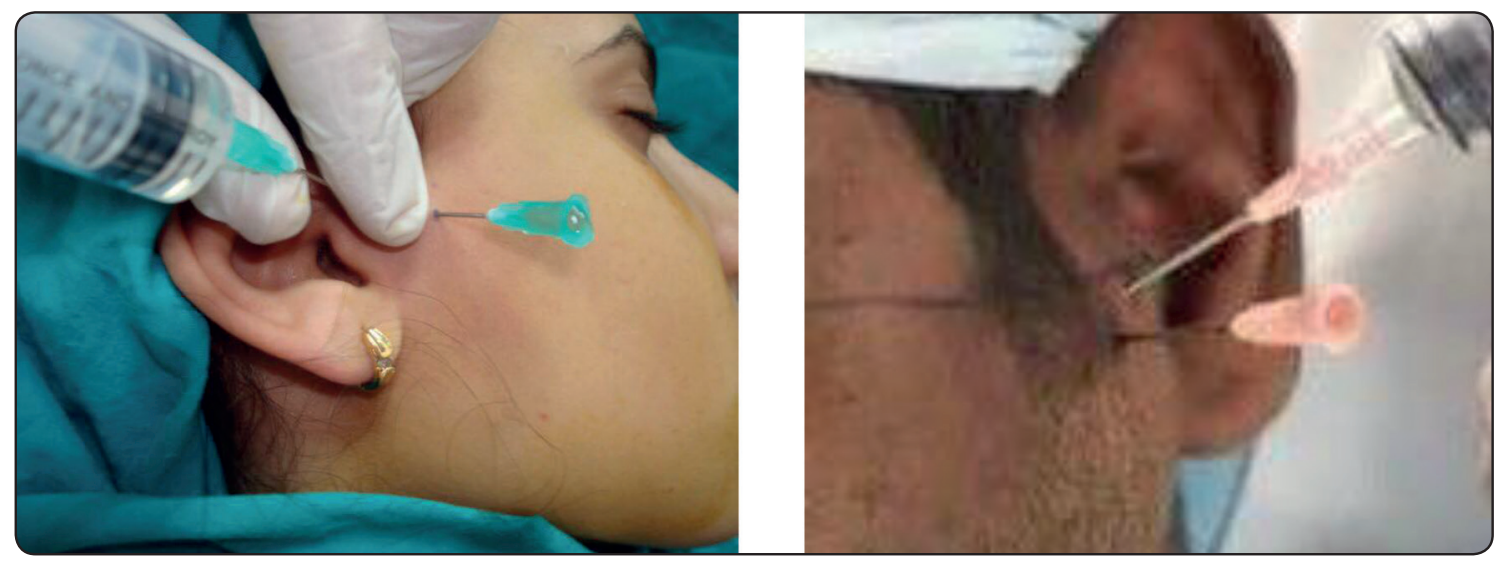

Fig. 3 (A) (B) Arthrocentesis for 2 patients in the control group .

All patients undergo the TMJ lavage procedure under local anesthesia using $100 \mathrm{ml}$ of Ringers lactate solution; the technique was standardized for all patients. The skin surface of the preauricular region was disinfected with povidone iodine solution. A canthal-tragal line was drawn and two points were marked to be used as the entry and exit for the lavage solution: the first at $10 \mathrm{~mm}$ anterior and $2 \mathrm{~mm}$ inferior; the second at $20 \mathrm{~mm}$ anterior and $6 \mathrm{~mm}$ inferior to the tragus on the drawn cantus-tragus line. The first point corresponded approximately with the posterior wall of the condylar fossa, which was also further confirmed manually by palpation over the area while manipulating the mandible in its range of motion. Auriculotemporal anesthesia was performed with (4\% articaine, adrenaline $1 / 100,000)$ which was also injected into the joint cavity. Two 20-gauge needles were placed at the entry and exit points for washing. The arthrocentesis was performed with $100 \mathrm{~mL}$ of Ringers lactate to eliminate the catabolites present in the synovial fluid. The mandible was then gently manipulated in the vertical, protrusive and lateral excursions to free the joint's compartments.

\section{PRP preparation \& injection technique}

PRP can be obtained using various methods, automated and/or manual, as reported in the literature. In this study, a one-step centrifugation procedure described by Anitua in 1999 was used (8). Intra-articular injections of PRP were preceded by the collection of peripheral blood from the patient ulnar vein using single-use, closed vacuum systems and glass tubes with sodium citrate as an anticoagulant to prevent early clotting. Mixing the collected blood with the citrate using rotational movements was done and even numbers of tubes were placed in a centrifuge rotor. The centrifugation parameters were 3,200 rpm and 12 minutes. After separation of the erythrocyte mass and the plateletpoor and platelet-rich plasma layers directly above the erythrocytes, the PRP was carefully aspirated into a separate syringe. $1 \mathrm{ml}$ of the prepared concentrate was injected into the TMJ utilizing the same reference points used in the arthrocentisis process (lateral cantus-tragus) after mixing with local anesthetic. We added local anesthesia to reduce postulated pain from the injection without reducing growth factor function or causing unwanted platelet activation. The entire procedure was performed under sterile conditions.

Also the injection technique adopted in the HA injection uses the same reference points as arthrocentisis process (lateral cantus-tragus). $1 \mathrm{~mL}$ of Low-molecular weight (ie, $600-\mathrm{kDa}$ ) HA (Hyalgan;Fidia, Abano Terme, Italy) was used. 


\section{Statistical Analysis}

All statistical analyses were carried out using SPSS (Statistical Package of Social Sciences, Chicago, IL, USA) for Windows software program version 17.0. A P-value of less than 0.05 was considered statistically significant. The results were expressed as the mean \pm standard deviation (SD). The mean changes in the parameters were calculated and the data used for intergroup comparisons were examined using the Mann-Whitney U test.

\section{RESULTS}

The final study sample consisted of 46 subjects with TMJ-OA in 38 patients, we lost 5 patients in the follow up period and they were excluded from the study. The mean age was 36.6 years with a range of 21 to 63 years. The majority of the patients were female $(80 \%)$. No statistically significant differences were observed in the baseline characteristics of the study groups for all of the study variables regarding mean age values, gender distributions and followup period (Table 1).

TABLE (1) Illustrates the comparisons of the baseline values for demographic features between the study groups.

\begin{tabular}{|c|c|c|c|c|}
\hline & $\begin{array}{c}\text { PRP } \\
\text { group }\end{array}$ & $\begin{array}{c}\text { HA } \\
\text { group }\end{array}$ & $\begin{array}{c}\text { Lavage } \\
\text { group }\end{array}$ & p-value \\
\hline Sample size & $\mathrm{n}=14$ & $\mathrm{n}=17$ & $\mathrm{n}=15$ & $\begin{array}{c}\text { Not } \\
\text { applicable }\end{array}$ \\
\hline $\begin{array}{c}\text { Age (years, mean } \\
\pm \text { SD) }\end{array}$ & $\begin{array}{c}37 \\
\pm 12.5\end{array}$ & $\begin{array}{c}36.2 \\
\pm 14.5\end{array}$ & $\begin{array}{c}34.2 \\
\pm 11.5\end{array}$ & 0.638 \\
\hline $\begin{array}{c}\text { Gender: Female/ } \\
\text { male }\end{array}$ & $11 / 3$ & $14 / 4$ & $12 / 2$ & 0.835 \\
\hline $\begin{array}{c}\text { Pain: VAS pre } \\
(0-10)\end{array}$ & $\begin{array}{c}8.15 \pm \\
0.64\end{array}$ & $\begin{array}{c}8.2 \\
\pm 0.41\end{array}$ & $\begin{array}{c}7.73 \\
\pm 0.93\end{array}$ & 0.485 \\
\hline $\begin{array}{c}\text { MMO pre (mm) } \\
\pm 4.65\end{array}$ & $\begin{array}{c}29.94 \\
\pm 5.73\end{array}$ & $\begin{array}{c}26.52 \\
\pm 5.02\end{array}$ & 0.528 \\
\hline Joint sounds & $\begin{array}{c}14 \\
(100 \%)\end{array}$ & $\begin{array}{c}17 \\
(100 \%)\end{array}$ & $\begin{array}{c}15 \\
(100 \%)\end{array}$ & $\begin{array}{c}\text { Not } \\
\text { applicable }\end{array}$ \\
\hline
\end{tabular}

Statistical analysis of our results in relation to both age and gender revealed the following:

\section{Regarding age:}

There was a direct relation with both the VAS and joint sounds:

The VAS relation was statistically significant ( $\mathrm{p}$ $<0.0001$ ) while the joint sound was not significant $(\mathrm{P}>0.05)$, there was an inverse relation with maximum mouth opening with age of the patient which was statistically significant $(p<0.0001)$

\section{Regarding gender:}

Statistically significant direct relation do exist in the VAS with females $(p<0.0001)$ and maximum mouth opening with males $(\mathrm{p}<0.0001)$, with no existing relation with joint sounds in both genders $(\mathrm{p}=0.137)$ (Table 2):

TABLE (2) Relations of age and sex with the outcome variables

\begin{tabular}{|c|c|c|}
\hline & Age & Gender \\
\hline VAS $(0-10)$ & $<0.0001^{* *}$ & $<0.0001^{* *}$ \\
\hline Joint sound & $0.172^{\mathrm{ns}}$ & $0.137^{\mathrm{ns}}$ \\
\hline MMO (mm) & $<0.0001^{* *}$ & $0.057^{*}$ \\
\hline
\end{tabular}

* significant $\cdot * *$ highly significant $\cdot$ ns $=$ non-significant

After 6 months, the 3 treatment techniques resulted in variable degree of clinical improvements for all the outcome variables in term of VAS scores, MMO and joint sounds, where pain and joint sounds decreased and MMO without pain increased significantly in all groups. the Intergroup comparisons of the out-come variables demonstrated no statistically significant differences in the mean changes between groups I and II for all VAS score, joint sound and MMO without pain $(\mathrm{P}>0.05)$, the clinical improvement in group I and group II was more than that occurred in group III (the control group) and was statistically significant 
$(\mathrm{p}<0.05)$. While at the end of the follow-up period after 12 months: the 3 groups vary in maintain these clinical improvements:

\section{Regarding Pain (VAS scores)}

After 6 months, Group III exhibited significantly greater median pain scores than Group I and group II (median pain scores: 7.0, 2.0, and 4.0, respectively). After 12 months, Group I exhibited significantly lower median pain scores than Group II (median pain score 1.0 with a range from 0 to 3.0).while group III was the higher in median pain values obtained. Regarding the changes over time in Group I, the pain values continued to decrease throughout the whole study period until the end of treatment. In Group II, there was a significant decrease in median pain score after 6 months (median pain score: 4.0 with a range from 3.0 to 5.0), but after 12 months, a significant increase in median pain score was observed (median pain score: 6.0 with a range from 4.0 to 7.0). However, at 12 months, the median pain score was significantly lower than that at the preoperative period. In Group III, after 6 months, there was no significant decrease in the pain levels, (median pain score: 9.0 with a range from 5.0 to 9.0). Also after 12 months, a significant increase in median pain score was observed ((median pain score: 10.0 with a range from 7.0 to 10.0). And this median pain score was significantly higher than that at the preoperative time (Table 3).

\section{Joint sounds}

There were no significant differences between the three groups after 6 and 12 months. Regarding the changes over time in Group I, there was a significant decrease in the prevalence of joint sounds after 6 months, At 12 months, a significantly lower prevalence of joint sounds compared to preoperative value, but this value was not significantly different from that at 6 months. In Group II, there was a significant decrease in the prevalence of joint sounds after 6 months and at 12 months, at 12 months; the prevalence of joint sounds was lower than the preoperative value. In Group III, there was no significant decrease in the prevalence of joint sounds after 6 and 12 months (Table 3 ).

\section{Regarding MMO:}

Group I exhibited a significantly higher median MMO than Group II while group III was the worst in the clinical improvement. Regarding the median MMO through all periods of follow-up. In Group

TABLE (3) Intragroup comparisons of outcome variables

\begin{tabular}{|c|c|c|c|c|c|}
\hline Variable & Group & Preopative & 6 months & 12 months & $\mathrm{P}$ value \\
\hline \multirow{3}{*}{$\begin{array}{c}\text { Pain } \\
\text { complaints } \\
\text { (VAS score) }\end{array}$} & GroupI: (PRP group) & $8 \pm 4.56$ & $2 \pm 1.08$ & $1 \pm 1.09$ & $0.0034 *$ \\
\hline & GroupII: (HA group) & $9 \pm 3.17$ & $4 \pm 2.86$ & $6 \pm 3.06$ & $0.0059 *$ \\
\hline & Group III: (Control group) & $8 \pm 4.63$ & $7 \pm 1.87$ & $10 \pm 4.74$ & $0.0038^{*}$ \\
\hline \multirow{3}{*}{$\begin{array}{l}\text { Joint sound } \\
\text { (VAS score) }\end{array}$} & GroupI: (PRP group) & $9 \pm 3.09$ & $6 \pm 2.96$ & $5 \pm 2.74$ & $0.00643 *$ \\
\hline & Group II: (HA group) & $9 \pm 4.39$ & $5 \pm 4.87$ & $6 \pm 6.05$ & $0.0031 *$ \\
\hline & GroupIII: (Control group) & $9 \pm 4.85$ & $7 \pm 2.56$ & $8 \pm 2.54$ & $0.71 \mathrm{~ns}$ \\
\hline \multirow{3}{*}{$\begin{array}{c}\text { Maximum } \\
\text { mouth } \\
\text { opening } \\
(\mathrm{MMO}) \\
(\mathrm{mm})\end{array}$} & Group I: (PRP group) & $33 \pm 7.05$ & $42 \pm 8.94$ & $44 \pm 9.51$ & $0.00043 *$ \\
\hline & $\begin{array}{l}\text { GroupII: } \\
\text { (HA group) }\end{array}$ & $34 \pm 6.93$ & $40 \pm 6.55$ & $37 \pm 7.65$ & $0.0059 *$ \\
\hline & GroupIII: (Control group) & $33 \pm 3.98$ & $38 \pm 5.64$ & $33 \pm 6.6$ & $0.68 \mathrm{~ns}$ \\
\hline
\end{tabular}


II and group III significant improvements were observed in the median MMO after 6 months then after 12 months, a statistically significant decrease in the median MMO was observed (40.0 and $37.0 \mathrm{~mm}$, respectively for group II ) and (38.0 and $33.0 \mathrm{~mm}$, respectively for group III) . However, at 12 months, the MMO was significantly greater than the preoperative value for group II and nearly returned to the preoperative value in group III (Table 3).

No complications related to the injections were observed during the treatment or follow-up period in all the groups of the study.

\section{DISCUSSION}

$\mathrm{OA}$ is a degenerative disorder of the synovial joints that affects the articular cartilage and subcondylar bone, In comparison to other joints, the TMJ has unique characteristics that are associated with the early appearance of TMJ-OA. Unlike long bones, the TMJ preserves its remodeling potential due to its proliferative mesenchymal cells, enabling accepted response to treatment at any age ${ }^{(9)}$. In the field of TMJ-OA practice, several approaches have been proposed over the years to manage symptoms; however TMJ-OA literature is characterized by some shortcomings regarding the level of knowledge on treatment effectiveness at the individual level.

When conservative methods such as medical treatment and occlusal splints fail to relieve pain or improve adequate jaw function, arthrocentesis (either alone or with other injections) became a primary tool as a minimally invasive treatment choice in the treatment of TMJ-OA, effectively filling the gap between failed conservative treatments and complex surgical procedures. A previous study on 36 patients with TMJ-OA found that arthrocentesis obviated the need for corrective surgery in $68.4 \%$ of the patients who do not responded to other nonsurgical treatment and were candidates for surgery. It significantly increased MMO and significantly decreased the subjectively assessed pain and dysfunction ${ }^{(6)}$.
This study aimed to compare the improvements in the joint symptoms and function in the groups who recieved PRP and the HA injections after arthrocentesis, one tried to remove the possible effects of arthrocentesis on the results observed in the two groups by making a third control group who underwent arthrocentesis alone with no agent injection. Pretreatment (baseline) bivariate associations between the study and predictor variables confirmed that there was no statistical difference between the predictor variables in age, gender, follow-up period, and baseline values of the outcome variables (Table 1). Therefore, any variable having the potential to affect the outcomes was eliminated at the beginning of the study, and the treatment strategies used for TMJ-OA remained the primary predictor variables. The out-comes of our study clearly showed that the application of PRP or HA injections into the TMJ following arthrocentesis resulted in promising functional recovery and benefits for patients who suffer from TMJ OA, regarding the arthrocentisis alone, despite arthrocentesis alone has been used widely for the treatment of internal TMJ problems, however, its effectiveness is temporary as it does not rehabilitate the micro-architecture of the TMJ.

The current results showed that there was no statistically difference in the amount of clinical improvement in both the PRP and the HA groups at the 6 month follow up, whereas both were superior than the group who received lavage only as the mere treatment, after 1 year, the best clinical outcome was in the group injected with PRP followed by the one injected with the HA injection. Overall, the clinical improvement occurred in patient with TMJOA in this study agrees with the claim that states injection of PRP into the joint spaces could improve and enhance the micro condition within the joint space and possibly prevent TMJ disease progression over long. Surprisingly, one think the better clinical improvement occurred in the PRP group compared to the HA one is attributed to the effect of the PRP on endogenous HA itself, as PRP aids in restoring 
the normal levels of endogenous HA which is considered as the main synovial fluid component and is dramatically decreased in the degenerative joint diseases, also PRP improves the HA biological properties ${ }^{(11)}$. We actually believe that both PRP and HA appears to complement each other.

The current results were coinciding with the postulation which assumed that the injection of some biological or non-biological agents, which have tissue recovery capacity into the TMJ may be helpful for the onset and the maintenance of the regenerating processes. Intra-articular injections of many different agents as PRP and HA have been postulated for treatment of TMJ-OA ${ }^{(9-12)}$. Recent studies assumed that arthrocentesis combined with intra-articular injections of various agents give superior results to arthrocentesis alone in management of TMJ-OA ${ }^{(12)}$. Additionally, it has been proposed that intra-articular injections may inhibit degenerative changes of cartilage and stimulate cartilage regeneration ${ }^{(12)}$.

Other studies that were conducted on other degenerative joints such as the knee support our results, Spakova et al treated knee osteoarthritis with PRP and hyaluronic acid in 120 patients and claimed significantly better results in the patients approached with PRP. Because no severe adverse events were observed by the investigators, the application of autologous PRP was reported as an effective and safe technique for the treatment of the starting phases of knee osteoarthritis ${ }^{(13)}$, also the study by Sampson et al showed improved function and decreased pain in 14 patients with knee OA 12 months after treatment with PRP injections ${ }^{(14)}$. The rationale for the use of PRP was that a great amount of growth factors that work synergistically are released from the activated platelets, these biologically active proteins stimulate the differentiation and proliferation of fibroblasts, chondrocytes, osteoblasts and mesenchymal stem cells.

Similar to our results, Hegab et al concluded that PRP give superior outcomes compared to
HA in terms of pain reduction and joint sounds in patients with TMJ OA, they applied three sessions of intra-articular PRP and Hyalgan but without $\operatorname{arthrocentesis}^{(15)}$.

In our study we used Hyalgan, as it is an injectable form of HA that has a low molecular weight with good penetrating action into the TMJ structures. The favourable outcomes we gained in the HA group are in agreement with the findings of the studies done by Manfredini D.et al., who stated that HA injections have a huge effects on the TMJ structures; They found great clinical improvements in MMO, TMJ pain, and masticatory efficiency during the treatment phase and follow-up period, they attributed the progress to the HA ability to enhance joint stabilization and joint space nutrition, also HA restore normal lubrication so decreased friction in the weared cartilage ${ }^{(16)}$.

Despite some controversy, there are many reports of HA having analgesic properties as a potential result of decreased both prostaglandin E2 production and ion channel sensitivity of mechanoreceptors. However, it is controversial whether intra-articular HA injection really modifies the progression of joint damage in OA. A protocol giving 5 weekly arthrocenteses plus HA injections has been proposed as an effective mean to manage symptoms of TMJ inflammatory-degenerative disease, and positive findings have been reported in case series of patients followed up for 1 year $^{(17)}$, the rationale for the use of $\mathrm{HA}$ in the treatment of TMJ-OA is that the direct injection of HA into the joint space permits the achievement of proper concentrations with low doses that favors greater permanence in the joint and therefore favors the therapeutic response

Contrary to what we found in the current study, another study by Guarda-Nardini et al., focused on 12 month follow-up of patients who had degenerative joint disease and underwent arthrocentesis and HA injection; they found that the short-term improvements in the symptoms of TMJ OA were maintained at the end of the 1 year follow-up ${ }^{(18)}$. 


\section{CONCLUSION}

The findings of the present study suggest that the application of PRP for the treatment of OA may be a promising and innovative procedure that can stimulate the repair of damaged tissues in TMJ-OA. It was more effective than HA injection. However, before suggesting this procedure to patients, more clinical trials are needed to confirm the conclusions.

\section{RECOMMENDATIONS}

Longer follow-up periods are strongly needed to support our findings, also further analysis of the synergistic actions of PRP and HA in the treatment of TMJ-OA will be very helpful.

\section{CONFLICT OF INTEREST}

None declared

\section{REFERENCES}

1. Comert Kilic S, Kilic N, Sumbullu MA: Temporomandibular joint osteoarthritis: cone beam computed tomography findings, clinical features, and correlations. Int J Oral MaxillofacSurg 44:1268, 2015.

2. Liu F, Steinkeler A. Epidemiology, diagnosis, and treatment of temporomandibular disorders. Dent Clin North Am 2013;57: 465-79.

3. Nitzan DW, Benoliel R, Heir G, Dolwick F: Pain and dysfunction of the temporomandibular joint. In: Sharav Y, Benoliel R (Eds). Orofacial pain and headache. London: Elsevier Health Sciences 149, 2008.

4. Sanders B: Management of internal derangements of the temporomandibular joint. SeminOrthod 1:244, 1995.

5. Lisa A. Fortier, Catherine H. Hackett, Brian J. Cole. The Effects of Platelet-Rich Plasma on Cartilage: Basic Science and Clinical Application. Operative Techniques in Sports Medicine Volume 19, Issue 3, pp 154-159, 2011.

6. Shi Z, Guo C, Awad M: Hyaluronate for temporomandibular joint disorders. Cochrane Database Syst Rev 1:CD002970, 2003.

7. Manfredini D, Rancitelli D, Ferronato G, et al: Arthrocentesis with or without additional drugs in temporomandibular joint inflammatory-degenerative disease: Comparison of six treatment protocols. J Oral Rehabil 39:245, 2012.
8. Anitua E. Plasma rich in growth factors: preliminary results of use in the preparation of future sites for implants. Int J Oral Max-illofac Implants 1999;14:529-35.

9. Comert Kilic S, Gungormus M, Sumbullu MA: Is Arthrocentesis Plus Platelet-Rich Plasma Superior to Arthrocentesis Alone in the Treatment of Temporomandibular Joint Osteoarthritis? A Randomized Clinical Trial. J Oral MaxillofacSurg 73:1473, 2015.

10. Møystad A, Mork-Knutsen BB, Bjørnland T (2008) Injection of sodium hyaluronate compared to a corticosteroid in the treatment of patients with temporomandibular joint osteoarthritis: a CT evaluation. Oral Surg Oral Med Oral Pathol Oral RadiolEndod105:e53-e60 16. Tsuruta A, Yamada.

11. Anitua et al., 2007; Anitua et al., 2012a; Wang-Saegusa et al., 2011; Sánchez et al., 2012; Vaquerizo et al., 2013.

12. Stoll ML, Good J, Sharpe T, Beukelman T, Young D, Waite PD, Cron RQ: Intra-articular corticosteroid injections to the temporomandibular joints are safe and appear to be effective therapy in children with juvenile idiopathic arthritis. J Oral Maxillofac Surg 70:1802, 2012.

13. Spakova T, Rosocha J, Lacko M, et al: Treatment of knee joint osteoarthritis with autologous platelet-rich plasma in comparison with hyaluronic acid. Am J Phys Med Rehabil 91:411, 2012.

14. Sampson S, Gerhardt M, Mandelaum B: Platelet rich plasma injection grafts for musculoskeletal injuries: A review. Curr Rev Musculoskelet Med 1:165, 2008.

15. Hegab AF, Ali HE, Elmasry M, Khallaf MG. Platelet-rich plasma injection as an effective treatment for temporomandibular joint oste-oarthritis. J Oral Maxillofac Surg 2015;73: 1706-13.

16. Manfredini D, Bonnini S, Arboretti R, Guarda-Nardini L. Temporomandibular joint osteoarthritis: an open label trial of 76 patients treated with arthrocentesis plus hya-luronic acid injections. Int J Oral Maxillofac Surg 2009;38:827-34.

17. Milam SB: TMJ osteoarthritis. In: Laskin DM, Greene CS, Hylander WL, eds. TMDs, an evidence-based approach to diagnosis and treatment, Chicago: Quintessence 105, 2006.

18. Guarda-Nardini L, Stifano M, Brombin C, Salmaso L, Manfredini D. A one-year case series of arthrocentesis with hyaluronic acid injections for temporomandibular joint os- teoarthritis. Oral Surg Oral Med Oral Pathol Oral Radiol Endod 2007;103: e14-22. 